\title{
Condições de saúde bucal de adolescentes em situação de vulnerabilidade social:
}

\section{revisão de literatura}

\author{
Oral health conditions of adolescents in socially vulnerable situations: literature review \\ Condiciones de salud bucal de adolescentes en situaciones de vulnerabilidad social: revisión de la \\ literatura
}

\author{
Flaviana Farias da Silva \\ ORCID: https://orcid.org/0000-0001-6282-3270 \\ Centro Universitário Fametro, Brasil \\ E-mail: fariasflaviana9@gmail.com \\ Francisca Érica Alves Cardoso \\ ORCID: https://orcid.org/0000-0001-5973-8673 \\ Centro Universitário Fametro, Brasil \\ E-mail: erica.alves.cardoso@gmail.com \\ Thiago Mendes de Lima \\ ORCID: https://orcid.org/0000-0001-6524-3271 \\ Centro Universitário Fametro, Brasil \\ E-mail: thiago.lima@fametro.edu.br \\ Gabriela de Figueiredo Meira \\ ORCID: https://orcid.org/0000-0002-7263-8711 \\ Universidades Federal de Santa Maria, Brasil \\ E-mail: Gabrielameira1@hotmail.com \\ Karina Alessandra Guimarães Barbosa \\ ORCID: https://orcid.org/0000-0003-4933-6421 \\ Associação Brasileira de Odontologia, Brasil \\ E-mail: karina.barbosa@fametro.edu.br
}

\begin{abstract}
Resumo
Introdução: A Organização Mundial da Saúde (OMS) considera adolescentes indivíduos com idade de 15 a 19 anos. É nesta fase que as mudanças marcantes acontecem e permanecem ao longo da vida. Há uma grande preocupação com corpo e com a aparência, incluindo nesse contexto a saúde bucal que também é parte do bem-estar individual. Objetivo: O presente trabalho tem como objetivo apresentar fatores que podem influenciar na saúde bucal de adolescentes entre 15 e 19 anos, em condição de vulnerabilidade social, por meio de uma revisão de literatura e nos resultados do Levantamentos de Saúde Bucal (SB Brasil) realizados nos anos 2003 e 2010. Metodologia: Trata-se de uma revisão bibliográfica com abordagem descritiva. Resultados e Discussão: Contudo, a situação social de vulnerabilidade associada a precária condição de vida, são fatores que deixam registros aparentes na boca, no sorriso desses adolescentes elevando os índices de cáries e perdas dentárias em adolescentes com baixa renda, baixo nível educacional, falta de acesso aos serviços de saúde bucal, associado à uma dieta cariogênica. Conclusão: Como consequência, a autoestima dos adolescentes que sofrem perda dentárias precoce é prejudicada pela má oclusão, causando falta de confiança, comprometimento nas atividades, desmotivação e dificuldade na comunicação.
\end{abstract}

Palavras-chave: Adolescência; Autoestima; Saúde bucal; Má oclusão.

\begin{abstract}
Introduction: The World Health Organization (WHO) considers adolescents aged 15 to 19 years. It is at this stage that marked changes take place and remain throughout life. There is great concern with the body and appearance, including in this context oral health, which is also part of individual well-being. Objective: This study aims to present factors that can influence the oral health of adolescents between 15 and 19 years old, in a condition of social vulnerability, through a literature review and the results of the Oral Health Surveys (SB Brasil) carried out in 2003 and 2010. Methodology: This is a literature review with a descriptive approach. Results and Discussion: However, the social situation of vulnerability associated with precarious living conditions are factors that leave apparent registers in the mouth, in the smile of these teenagers, increasing the rates of tooth decay and tooth loss in teenagers with low income, low educational level, lack access to oral health services, associated with a cariogenic diet. Conclusion: As a consequence, the self-esteem of adolescents who suffer early tooth loss is impaired by malocclusion, causing a lack of confidence, compromised activities, lack of motivation and communication difficulties.
\end{abstract}

Keywords: Adolescence; Self esteem; Oral health; Malocclusion. 


\begin{abstract}
Resumen
Introducción: La Organización Mundial de la Salud (OMS) considera adolescentes de 15 a 19 años. Es en esta etapa que se producen cambios marcados y se mantienen a lo largo de la vida. Existe una gran preocupación por el cuerpo y la apariencia, incluida en este contexto la salud bucal, que también forma parte del bienestar individual. Objetivo: Este estudio tiene como objetivo presentar los factores que pueden influir en la salud bucal de adolescentes entre 15 y 19 años, en condición de vulnerabilidad social, a través de una revisión de la literatura y los resultados de las Encuestas de Salud Bucal (SB Brasil) realizadas en 2003. y 2010. Metodología: Se trata de una revisión de la literatura con enfoque descriptivo. Resultados y Discusión: Sin embargo, la situación social de vulnerabilidad asociada a las precarias condiciones de vida son factores que dejan aparentes registros en la boca, en la sonrisa de estos adolescentes, aumentando las tasas de caries y pérdida de dientes en adolescentes de bajos ingresos, bajo nivel educativo. nivel, falta de acceso a servicios de salud bucal, asociado a una dieta cariogénica. Conclusión: Como consecuencia, la autoestima de los adolescentes que sufren una pérdida dentaria prematura se ve afectada por la maloclusión, provocando falta de confianza, actividades comprometidas, falta de motivación y dificultades de comunicación.
\end{abstract}

Palabras clave: Adolescencia; Autoestima; Salud bucal; Maloclusión.

\title{
1. Introdução
}

A situação social de vulnerabilidade associada a precária condição de vida, são fatores que deixam registros aparentes na boca, no sorriso das pessoas. Condição essa rotulada pela pobreza, descaso e fracasso que, ao passar dos anos, o corpo responde fisicamente deixando suas marcas na face e psicologicamente, marcando também o modo de convivência. A dentição, quase sempre, exprime a oportunidade que o cidadão teve de superar ou não suas mazelas sociais (Moreira et al., 2007).

Uma educação deficiente e o contexto social que estão inseridos podem levar alguns jovens a não ter acesso a saúde. Nesta fase da vida, as modificações hormonais e uma dieta rica em alimentos cariogênicos alteram a microbiota bucal contribuindo para o predomínio de cárie e doenças periodontais (Araújo et al., 2017). Pesquisas mostram que no período da adolescência a patologia que mais afeta a cavidade bucal devido a frequente ingestão de alimentos com alto teor de açúcar e devido à ausência ou correta higiene bucal é a cárie (Sarmento et al., 2020).

Além da dieta, a saúde bucal também é prejudicada por fatores socioeconômicos que contribuem para um índice de cárie elevado em jovens de classe baixa pela falta de acesso a tratamento odontológico (Sarmento et al., 2020). A autoestima dos jovens que sofrem perda dentárias precoce é prejudicada causando falta de confiança, comprometimento nas atividades, desmotivação e dificuldade na comunicação. Das desordens na saúde bucal dos jovens que ocorrem com mais frequência podemos citar a má oclusão devido a perda precoce dos dentes (Lunardelli et al., 2016).

Em muitos casos as doenças periodontais e a cárie se agravam devido à falta de participação fundamental dos pais na vida dos jovens refletindo de forma negativa na saúde bucal do adolescente e deixando-os mais vulneráveis (Sarmento et al., 2020). As consequências influenciam de modo abrangente na vida dos jovens, incluindo a capacidade de desempenho de atividades cotidianas até mesmo à dificuldade de comunicação, relacionamento e aprendizado. O convívio diário com a dor e o desconforto limitam o consumo de alimentos e restringem o nicho de convivência afetando o desenvolvimento emocional desse grupo social. (Lunardelli et al., 2016).

Não se trata apenas de contabilização para censo demográfico. Pessoas de baixa renda que possuem sua condição social registrada em âmbito nacional naquilo que demonstra a situação de vida de milhares de brasileiros, possuem uma história de vida e uma realidade marcada pela dificuldade. Crianças e adolescentes em condição bucal desfavorável estendem ainda mais sua exclusão. Se não há como comer, não há como trabalhar, não há como cuidar da saúde tampouco cuidar de si próprio. (Moreira et al., 2004).

A presença da cárie dentária, segundo a literatura, tem interferido de forma psicológica na vida de crianças e adolescentes, pois os efeitos biológicos que causam desconforto são refletidos na forma comportamental destes jovens. 
(Fernandes et al., 2013). É nesta fase que as mudanças marcantes acontecem e permanecem ao longo da vida. Comportamentos positivos podem ser adquiridos, assim como o negativismo, o comportamento antissocial e delinquência. (Lunardelli et al., 2016).

No Brasil, há estudos relacionados à saúde bucal e autoestima de adolescentes, se destacando os levantamentos epidemiológicos nacionais conduzidos pelo Ministério da Saúde, elaborados em 2003 e 2010 . O presente trabalho tem como objetivo apresentar fatores que podem influenciar na saúde bucal de adolescentes entre 15 e 19 anos, em condição de vulnerabilidade social, por meio de uma revisão de literatura e com base nos resultados do SB Brasil 2003 e 2010 , na Região Norte do Brasil.

\section{Metodologia}

O presente estudo efetivou-se através de pesquisa descritiva, na qual propôs a identificação, registro e análise das características, fatores ou variáveis que se relacionam com o fenômeno ou processo (Perovano, 2014). Dessa forma, embasada em referências bibliográficas e científicas, relatando Condições de saúde bucal de adolescentes em situação de vulnerabilidade social

A presente pesquisa é do tipo revisão bibliográfica contando com a análise crítica de artigos publicados em periódicos científicos entre os anos de 2011 a 2021. As bases de dados e periódicos eletrônicos utilizadas para a seleção dos mencionados artigos foram: SciELo, Pubmed e Lilacs. Os critérios de exclusão foram artigos de língua estrangeira e que não versassem, especificamente, a respeito da aplicação da saúde bucal. Já os critérios de inclusão foram artigos em língua portuguesa e aqueles que envolvesse, especificamente, aplicação das metodologias da saúde bucal.

Além disso, é importante destacar que os descritores utilizados na busca das referências foram: Adolescência; Autoestima; Saúde Bucal; má oclusão. Com isso, todos os estudos foram devidamente analisados, selecionados de acordo com o tema apresentado, título e conteúdo para a construção do estudo. 
Figura 1: Fluxograma de seleção dos estudos incluídos na revisão

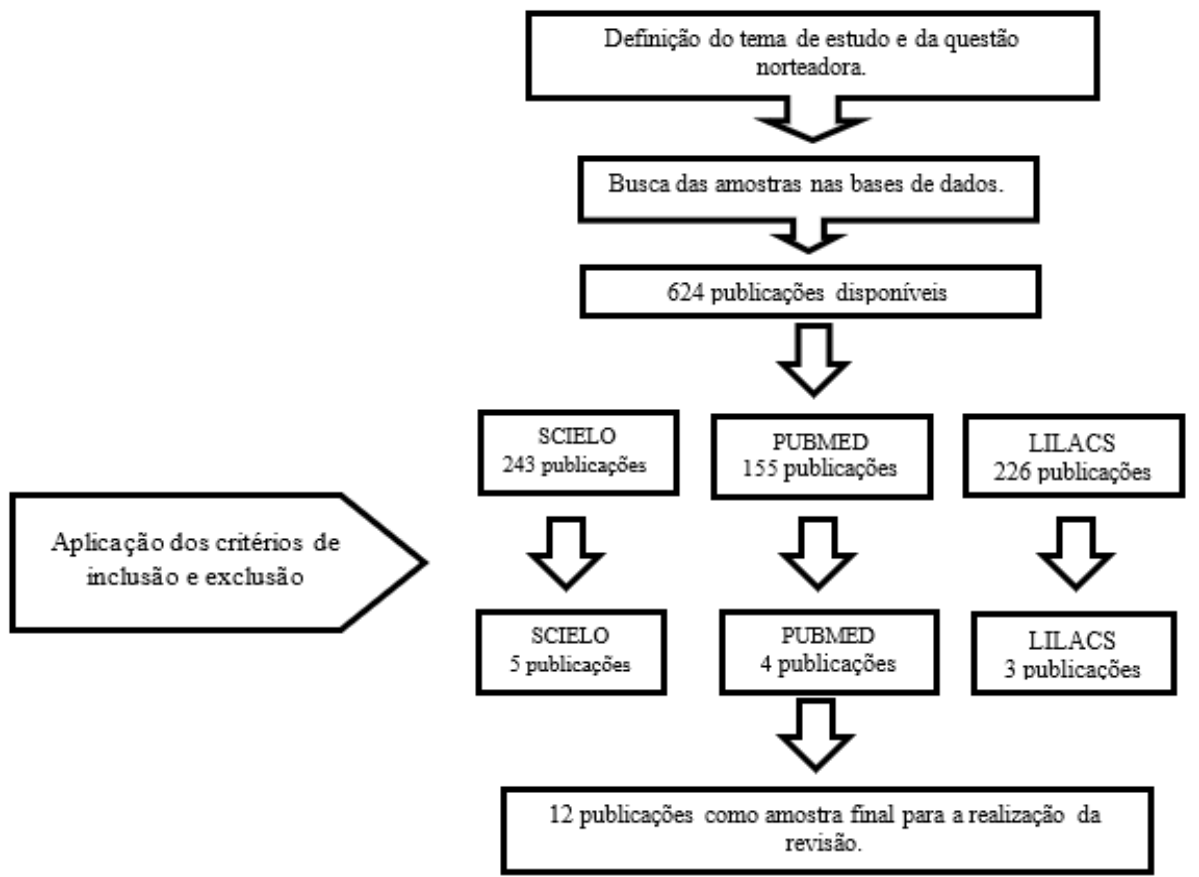

Fonte: Autoria própria.

\section{Resultados e Discussão}

Após as buscas através do cruzamento dos descritores nas bases indexadas: "adolescencia", "auto-estima”, "saúde bucal”, "má oclusão", foram resultados um total de 624 estudos.

Dos estudos resultantes, um total de 243 foram encontrados na base de dados Scielo, dos quais apenas 145 apresentavam o texto completo disponível, e 47 foram publicados nos últimos dez anos, resultando após análise crítica um total de 5 artigos que respondem à questão norteadora desse estudo.

Ao que se refere à base de dados Lilacs, após o cruzamento dos descritores foram encontrados um total de 226 estudos, dos quais apenas 197 estudos apresentavam o texto completo e disponível, e apenas 51 estudos foram publicados nos últimos dez anos obedecendo os critérios previamente estabelecidos, respondendo assim, após uma análise crítica 3 artigo à questão norteadora desse estudo.

Ao que se refere à base de dados Pubmed, após o cruzamento dos descritores foram encontrados um total de 155 estudos, dos quais apenas 103 estudos apresentavam o texto completo e disponível, e apenas 45 estudos foram publicados nos últimos dez anos obedecendo os critérios previamente estabelecidos, respondendo assim, após uma análise crítica 4 artigo à questão norteadora desse estudo.

Dessa forma, totalizaram um total de 12 estudos que iram compor esse estudo, os quais após análise crítica constatouse que respondem à questão norteadora do estudo aqui proposto, apresentados a partir do corpus desse estudo. 
Quadro 1. Corpus do Estudo.

\begin{tabular}{|c|c|c|c|c|}
\hline $\mathbf{N} \% \mathbf{A N O}$ & TITULO DO ESTUDO & PERIÓDICO & AUTORES & BASE DE DADOS \\
\hline 2020 & $\begin{array}{l}\text { Gengivitis influences oral health-related } \\
\text { quality of life in adolescents: findings from a } \\
\text { cohort study. }\end{array}$ & $\begin{array}{l}\text { Revista Brasileira de } \\
\text { Epidemiologia }\end{array}$ & Ortiz, F. R., et al. & Lilacs \\
\hline 2020 & $\begin{array}{l}\text { Prevalência de cárie dentária em crianças em } \\
\text { condição de vulnerabilidade social. }\end{array}$ & $\begin{array}{l}\text { Revista } \\
\text { Multidisciplinar e da } \\
\text { Psicologia }\end{array}$ & Vilar, M. O. & Pubmed \\
\hline 2020 & $\begin{array}{l}\text { Desafios da educação em saúde bucal na } \\
\text { adolescência. }\end{array}$ & REAOdonto & $\begin{array}{l}\text { Sarmento, M. G. S., } \\
\text { Santos O. A \& Lima M. } \\
\text { M. }\end{array}$ & Lilacs \\
\hline 2020 & $\begin{array}{l}\text { Persistem iniquidades sociais na distribuição } \\
\text { da cárie dentária em adolescentes } \\
\text { maranhenses? Contribuições de um estudo } \\
\text { de base populacional. }\end{array}$ & $\begin{array}{l}\text { Revista Digital da } \\
\text { Academia Paraense } \\
\text { de Odontologia. }\end{array}$ & Sousa, F. S., et.al. & Pubmed \\
\hline 2019 & $\begin{array}{l}\text { O impacto das doenças bucais de } \\
\text { adolescentes na rotina de famílias de } \\
\text { diferentes classes socioeconômicas. }\end{array}$ & $\begin{array}{l}\text { Rev. J Health Sci } \\
\text { Inst. Araçatuba-SP }\end{array}$ & Martins, R. J., et al. & Scielo \\
\hline 2019 & $\begin{array}{l}\text { Má oclusão e vulnerabilidade social: um } \\
\text { estudo representativo de adolescentes de } \\
\text { Belo Horizonte, Brasil. }\end{array}$ & $\begin{array}{l}\text { Rev. Ciência e } \\
\text { Saúde Coletiva. }\end{array}$ & Martins, L. P., et al & Scielo \\
\hline 2018 & $\begin{array}{l}\text { Prevalência e características da doença } \\
\text { periodontal em adolescentes: revisão de } \\
\text { literatura. }\end{array}$ & REAOdonto & Torres-Neto, J. R., et al. & Pubmed \\
\hline 2018 & $\begin{array}{l}\text { ONG Turma do Bem: Reabilitando Sorrisos } \\
\text { e Transformando Vidas. Relato de caso. }\end{array}$ & $\begin{array}{l}\text { Revista Digital da } \\
\text { Academia Paraense } \\
\text { de Odontologia. }\end{array}$ & $\begin{array}{l}\text { Andrade, M. N., Silva } \\
\text { W.M., Neto, L.A., \& } \\
\text { Pereira S. V.R. }\end{array}$ & Scielo \\
\hline 2018 & $\begin{array}{l}\text { Saúde bucal de adolescentes rurais } \\
\text { quilombolas e não quilombolas: um estudo } \\
\text { dos hábitos de higiene e fatores associados. }\end{array}$ & REAOdonto & Silva, E. K. P., et al. & Pubmed \\
\hline 2017 & $\begin{array}{l}\text { Fatores individuais associados à má-oclusão } \\
\text { em adolescentes. }\end{array}$ & $\begin{array}{l}\text { Rev. Ciência \& } \\
\text { Saúde Coletiva. }\end{array}$ & Rebouças, A. G., et al. & Lilacs \\
\hline 2017 & $\begin{array}{l}\text { Prevalência de cárie dentária, autopercepção } \\
\text { e impactos em saúde bucal em adolescentes } \\
\text { na ilha do Marajó - Pará. }\end{array}$ & $\begin{array}{l}\text { Revista Digital da } \\
\text { Academia Paraense } \\
\text { de Odontologia. }\end{array}$ & Araújo, M. V. A., et al. & Scielo \\
\hline 2016 & $\begin{array}{l}\text { Autoestima e carie dentaria em adolescentes: } \\
\text { um estudo seccional. }\end{array}$ & $\begin{array}{ll}\text { Rev } & \text { Odontol } \\
\text { UNESP }\end{array}$ & Lunardelli, S. P., et al & Scielo \\
\hline
\end{tabular}

Fonte: Autoria própria.

No Brasil, a desigualdade social e de renda provocam a concentração dos níveis de pobreza em grande parte da população, onde os menos favorecidos possuem acesso restrito à saúde e como consequência, uma baixa qualidade de vida. Este desequilíbrio socioeconômico que gera a escassez de recursos básicos, também marca as condições de saúde bucal dessas famílias, onde aqueles com maior poder aquisitivo garantem os melhores tratamentos, sejam eles preventivos ou curativos, e os menos favorecidos permanecem vulneráveis e às margens do acesso aos serviços de saúde. Os adolescentes, em situação de vulnerabilidade social, por não possuírem acesso aos materiais básicos para uma higiene oral adequada, apresentam os maiores níveis de doenças bucais, pois nesses casos, outras prioridades, como alimentar-se, por exemplo, são consideradas mais 
importantes. E como resultado, uma experiência ruim e presente em quase todos os casos é o convívio com a dor (Martins $e$ al., 2019).

Segundo a Organização Mundial da Saúde (OMS) a adolescência compreende a faixa etária de 15 a 19 anos, período seguinte à infância, marcado pela puberdade, onde ocorrem muitas mudanças corporais e mentais. Nesta fase, é onde a identidade e o padrão de comportamento tomam forma mais definida e se tornam mais acentuados. Nesse momento, a aceitação social, preocupação com a imagem e aparência estão em alta, principalmente pela cobrança interna e externa do adolescente em atingir os padrões impostos pela sociedade e é nesse momento que a saúde bucal ganha sua real importância. Contudo, algumas alterações podem ser mais difíceis quando o contexto social em que o sujeito está inserido incluem as situações de desigualdade social, pobreza e estilo de vida (Araújo et al., 2017).

A cárie dental é uma doença decorrente da relação entre os micro-organismos bucais e o hospedeiro, tendo como maior aliado o fator tempo e dieta pois estes auxiliam no processo de desmineralização dos dentes. Contudo, os fatores que realmente se destacam no surgimento da doença, estão intimamente ligados à dificuldade de acesso a tratamentos odontológicos e à água potável, dieta precária, higiene oral insuficiente e inadequada. Além de que, o contexto social de vulnerabilidade torna o surgimento da cárie dental, ainda mais frequente nos grupos populacionais com baixas condições econômicas (Vilar et al., 2020).

Nos dias de hoje ainda existem jovens que não possuem acesso à saúde e em sua grande maioria, pela escassez de recursos financeiros ou até mesmo por falta de motivação e orientação de seus responsáveis. Além disso, a alimentação rica em itens cariogênicos, coloca em destaque a presença da cárie e da doença periodontal como as doenças bucais mais prevalentes nesta fase, visto que o consumo é excessivo e realizado fora de casa, sem a supervisão dos pais. Além disso, adolescentes de classe baixa, não possuem acesso a tratamentos odontológicos na mesma proporção que necessitam, o que, degrada ainda mais a saúde bucal (Sarmento et al., 2020).

Levantamentos epidemiológicos sobre doenças periodontais mostram índices elevados de sangramento gengival e cálculo dental em variadas classes sociais e diferentes faixas etárias. Outros estudos, realizados no Brasil, entre os anos de 2003 e 2008, constatou que adolescentes de regiões mais pobres, com menos recursos, apresentaram alta taxa de sangramento gengival e também de cálculo dental. A higiene oral de qualidade associada a condições favoráveis de acesso à saúde, são marcos importantes que podem diminuir essa prevalência (Torres Neto et al., 2018).

A relação dos aspectos socioeconômicos e o nível de escolaridade com a baixa condição de saúde bucal, refletem também no alto índice de má oclusão severa. Muitas perdas de dentes anteriores e posteriores acontecem por cárie, facilitando o quadro de má-oclusão, passando a ser, acima de tudo, um marcador de exclusão social. Também, o nível de escolaridade contribui, de forma direta para que se tenha boas condições de saúde bucal, pois esses conhecimentos levam o indivíduo a reconhecer a necessidade da busca pela assistência odontológica (MartinS et al., 2019).

O levantamento de dados realizado pelo projeto SB Brasil no ano de 2004 mostrou que cerca de 14\% dos adolescentes brasileiros nunca compareceram ao consultório odontológico. O serviço público foi o mais procurado para atendimento, mostrando que, em média, 55\% dos brasileiros realizaram sua consulta através do Sistema Único de Saúde (SUS), fato que pode ser comparado ao grande número de adolescentes interrogados frequentarem escolas públicas. As consultas de manutenção e rotina foram as mais relatadas pelos entrevistados, alcançando aproximadamente $34 \%$ enquanto a experiência de dor, em volta de $31 \%$. Notou-se ainda que a região Sul foi a que apresentou a menor taxa de indivíduos não assistidos, cerca de $5 \%$, enquanto na região Nordeste e Norte essas taxas alcançaram, ao redor de $22 \%$ e $17 \%$, respectivamente, demostrando desigualdades entre as regiões brasileiras (Brasil, 2004).

Quanto à necessidade de tratamento, cerca de 55\% necessitavam restaurar uma superfície dentária e 24\% duas ou mais superfícies. Das alterações periodontais, a presença de cálculo e sangramento foram as que mais apresentaram números 
expressivos, com números em torno de 34\% e 18\% respectivamente. Nota-se aqui que nos entrevistados com faixa etária entre 35 a 44 anos, a presença de sangramento mostrou uma grande redução, chegando a aproximadamente $10 \%$ e a presença de cálculo um aumento, com números por volta de 47\%. As anormalidades dentofaciais, nos jovens, representaram, em média $59 \%$, sendo mais presente na região Sul, em torno de 64\% (Brasil, 2004).

O levantamento de dados realizados pelo projeto SB Brasil de 2010, mostrou que muita coisa mudou e outras permaneceram como antes em relação à pesquisa realizada em 2004. A visita ao consultório odontológico apresentou um leve declínio quando comparado ao visto anteriormente, representando cerca de $17 \%$. A região Norte foi a que apresentou a maior taxa de não visitas do país, com aproximadamente $66 \%$. O SUS ainda foi o mais procurado para realizar de atendimento, com taxa aproximada de 64\%. Nota-se aqui que o atendimento em serviço privado sofreu aumento chegando a $26 \%$. O motivo principal passou a ser a prevenção, com aproximadamente $28 \%$ e a extração em segundo lugar, com cerca de $26 \%$. Foram incluídos ainda dados de renda familiar entre as regiões brasileiras. É visto que aproximadamente 51\% da população brasileira apresenta renda de 501,00 a 1500,00 reais, o que explica grande parte da procura odontológica advir do serviço público, principalmente quando se verifica que, apenas cerca de 19\% da população, possui renda de 1501,00 a 1500,00 reais. Quando se verifica rendas mais baixas de $\mathrm{R} \$ 251,00$ a $\mathrm{R} \$ 500,00$ a região norte e nordeste apresenta as maiores taxas, com aproximadamente $20 \%$ (Brasil, 2010).

Das necessidades de tratamento, houve uma grande redução na restauração de uma superfície entre os adolescentes, alcançando em média 4\% entre as regiões brasileiras, sendo a maior na região norte com cerca de $8 \%$. Nas alterações periodontais, quando observado a população de 15 a 19 anos, houve um aumento quanto às taxas mostradas em 2004, com o índice de sangramento de aproximadamente 34\% e índice de cálculo de 36\%. Das condições de oclusão, a oclusopatia definida, em 2010, representou cerca de 20\%, enquanto a forma severa e muito severa, aproximadamente 10\% e $8 \%$. Ademais, a pesquisa mostrou que em volta de $37 \%$ da população residente na região Norte não está satisfeita com suas condições de saúde bucal. A avaliação de impacto de condições bucais na vida diária trouxe dados interessantes. A dificuldade na alimentação foi o impacto mais listado, seguido pelo aspecto emocional, contato social, realização de higiene oral, sorrir e dormir (Brasil, 2010).

O estado da saúde bucal das pessoas possui relação direta com o contexto socioeconômico no qual estão inseridas e se estabelecem desde a infância até a vida adulta. Araújo et al., (2017), afirma que a adolescência é um dos períodos de grandes e importantes mudanças do ser humano, visto que, nesse momento, a personalidade, o modo de agir e pensar, a ações e reações às adversidades da vida, são aqui, formadas. Para os autores, é nessa fase que saúde bucal deveria adquirir sua verdadeira importância, pois está diretamente ligada ao contentamento, a satisfação e felicidade.

Em consonância, Martins et al., (2019) demonstrou que as doenças bucais geram uma repercussão negativa na vida das crianças e adolescentes, causando problemas na socialização, autoestima, no comportamento, rendimento escolar e principalmente nas atividades básicas do dia-a-dia como falar, engolir, mastigar e dormir, impedindo que esses adolescentes tenham o mínimo de bem-estar. Tais consequências estão de acordo com os dados coletados pela SB Brasil (2010) em que as variáveis: dificuldade para comer, escovar os dentes, alteração do estado emocional e vergonha ao sorrir foram os impactos mais afirmados por adolescentes de 15 a 19 anos, representando respectivamente: $20,7 \%, 16,5 \%, 14,6 \%$ e $12,9 \%$.

A cárie dental é uma doença abrangente que possui uma evidente associação com as condições socioeconômicas de adolescentes. Sousa et al., (2020), em sua pesquisa com crianças vulneráveis de idades entre 3 a 14 anos, concluiu que os piores indicadores da doença cárie foram identificados em localidades onde não se tem acesso ao saneamento básico, causando o que se chama de "polarização" - em pequenos grupos de indivíduos, geralmente aqueles que não possuem acesso a serviços odontológicos ocorre uma grande concentração da cárie. 
Em conformidade, Lunardelli et al., (2016) mostrou que as regiões Norte e Nordeste apresentam as menores coberturas de água fluoretada do país (19\%) enquanto as regiões, Sul e Sudeste, apresentam taxas de $95 \%$ e 83,9\%, respectivamente, sendo as únicas que atingiram as metas da Organização Mundial de Saúde em relação à redução do índice de cárie. Tais fatos mostram a relação entre as condições de saneamento e a redução no índice da doença cárie.

Dados do SB Brasil de 2004 e 2010, mesmo com a criação do programa "Brasil Sorridente" em 2004 e a inclusão da equipe de saúde bucal no programa Estratégia Saúde da Família (ESF), afirmam que as regiões Norte e Nordeste sofreram um aumento discreto na prevalência da cárie, sendo classificada de nível moderado. Além do mais, a pesquisa demonstrou que as regiões citadas, são as que possuem a menor média de renda familiar assim como a cobertura de abastecimento de água, comparada às demais regiões do país.

Quanto às condições periodontais, os dados contidos no Projeto SB Brasil (2010) demostram que os adolescentes de 15 a 19 anos, de baixa renda, apresentam alto índice de sangramento e cálculo gengival, sendo a região Norte aquela que possui as maiores taxas, $51 \%$ e $57,2 \%$, respectivamente, além da presença de bolsa rasa com $21,4 \%$, constatando assim, que condições socioeconômicas influenciam, também, nas doenças do periodonto, visto que, as regiões mais pobres são as que apresentam os maiores índices.

Torres Neto et al., (2018) complementam afirmando que a forma mais eficaz de se evitar a doença periodontal na adolescência é a realização contínua de uma higiene oral adequada, com o uso da escova dental acompanhada de dentifrício com flúor, para que haja a redução do biofilme. Além disso, se faz necessária a raspagem e alisamento radicular, de forma regular. Todavia, por se tratar de uma doença multifatorial, é necessário considerar a presença de outros fatores de risco, como alterações sistêmicas, comportamentais e socioeconômicas do adolescente.

Ortiz et al., (2020) relataram que a gengivite contribui, de forma negativa, na qualidade de vida dos adolescentes, afetando o comportamento psicossocial desses indivíduos, prejudicando suas relações sociais e autoestima. No estudo, os autores demonstraram que vermelhidão e inchaço das gengivas são os fatores que muito incomodam os adolescentes, pois por meio do sorriso, essa condição fica bastante evidenciada. Além disso, os participantes com renda familiar mais baixa e que suas mães possuíam baixo nível escolar, apresentaram as pontuações mais altas. E isso está relacionado ao fato da gengivite ser uma consequência da qualidade da higiene oral, muitas vezes inexistente ou precária àqueles de baixa renda e também baixa escolaridade.

Além disso, existe ainda a presença das má-oclusões, que, por meio da pesquisa realizada no o estudo da SB Brasil de 2004, foi constatado que cerca de $58 \%$ da população brasileira, apresentava algum problema de oclusão, onde, desse percentual, 32\% eram adolescentes acometidos por condições severas e em alguns casos, incapacitantes. Já no estudo de 2010, essa porcentagem reduziu alcançando números de 17,5\% dos mesmos parâmetros (ORTIZ et al., 2020).

Rebouças et al., (2017), complementam, afirmando que o maior fator de risco da má-oclusão é a perda dentária, pois quando essa perda acorre de forma precoce, as migrações dentárias acontecem e alteram a oclusão dos indivíduos. Os mesmos autores afirmam que a redução dos índices de alterações oclusais pode estar relacionada ao fato da redução de perda dentária, pois houve uma queda que partiu de 0,89 em 2004 para 0,38 em 2010. Em 2004 o país não dispunha de nenhuma política voltada para melhoria da saúde bucal por meio de programas sociais que cobrisse parte da necessidade daqueles de classe social baixa. Já em 2010, contava-se com a presença do Programa Nacional de Saúde Bucal, alcançando comunidades carentes e desassistidas e com isso houve uma redução do número de dentes perdidos. Outro indicador analisado foi a renda familiar. Este indicador, sugere que indivíduos com perda dentária e severidade da má oclusão são mais excluídos socialmente e possuem menos oportunidades de vida.

Martins et al., (2019), colaboram com o estudo e acrescentam que vulnerabilidade social e má-oclusão acentuada, representa uma desvantagem social, pois a estética, em especial, de dentes anteriores, interfere nas relações sociais, no bem- 
estar psicológico e comprometem a inter-relação entre os adolescentes pois a boa aparência é um importante aliado no estabelecimento de vínculos humanos.

Os autores demonstraram que a vulnerabilidade social tem relação direta com a capacidade dos pais em ofertar, aos seus filhos, ainda na infância, acesso a atendimento adequado e a bons hábitos de escovação. Relacionaram, os autores, que desta forma, a saúde bucal do adolescente ficará comprometida e que não somente o sorriso será cessado. Tantas outras dificuldades serão vivenciadas no decorrer de todas as fases da vida.

\section{Conclusão}

Com o presente estudo, conclui-se que fatores relacionados aos aspectos socioeconômicos, deficiência na escolaridade, dieta rica em alimentos com alto teor de açúcar e precárias condições de vida estão associados diretamente a baixa condição de saúde bucal dos adolescentes que como consequência sofrem perda dentárias precoces influenciando na qualidade de vida, capacidade de desempenho de atividades cotidianas, dificuldade de comunicação, relacionamento e aprendizado. Notou-se ainda, que o hábito de ir ao dentista, em regiões de vulnerabilidade social é uma realidade distante e que medidas preventivas para recuperação da saúde bucal, devem ser realizadas com maior frequência para que haja diminuição do número de adolescentes com perda precoce dos dentes e melhor condições de saúde bucal nesta região e em todo o Brasil.

\section{Referências}

Andrade, M. N., Silva W.M., Neto, L.A., \& Pereira S. V.R. (2018). ONG Turma do Bem: Reabilitando Sorrisos e Transformando Vidas. Relato de caso. Universidade Estadual da Paraíba, UEPB, Campina Grande-PB.

Araújo, M. V. A., et al. (2017). Prevalência de cárie dentária, autopercepção e impactos em saúde bucal em adolescentes na ilha do Marajó - Pará. Revista Digital da Academia Paraense de Odontologia. Belém/PA, v.1(1).

Brasil. (2004). Ministério da Saúde. Secretaria de Atenção à Saúde. Departamento de Atenção Básica. Projeto SB Brasil 2003. Condições de saúde bucal da população brasileira 2002-2003: resultados principais. Brasília: Ministério da Saúde.

Brasil. (2011). Ministério da Saúde. Portaria GM nº 2488 de 21 de outubro de 2011. Política Nacional de Atenção Básica.

Davoglio, R. S., et al. (2008). Fatores associados a hábitos de saúde bucal e utilização de serviços odontológicos entre adolescentes. Porto Alegre, RS.

Lunardelli, S. P., et al. (2016). Autoestima e carie dentaria em adolescentes: um estudo seccional. Rev Odontol UNESP.

Machado, F. C. A., et al. (2015). Proposição de indicadores para vigilância da saúde bucal de adolescentes. Natal RN Brasil.

Martins, R. J., et al. (2019). O impacto das doenças bucais de adolescentes na rotina de famílias de diferentes classes socioeconômicas. Rev. J Health Sci Inst. Araçatuba-SP, 37(1), 20-25.

Martins, L. P., et al. (2019). Má oclusão e vulnerabilidade social: um estudo representativo de adolescentes de Belo Horizonte, Brasil. Rev. Ciência e Saúde Coletiva. Belo Horizonte-MG, 24(2), 393-400.

Moreira, T.P., Nuto, S.A.S., \& Nations, M.K. (2004). Confrontação cultural entre cirurgiões-dentistas e a experiência de usuários de baixa renda em Fortaleza - CE. Saúde Debate, 66, 58-67.

Moreira, T. P., et al. (2007). Dentes da desigualdade: marcas bucais da experiência vivida na pobreza pela comunidade do Dendê, Fortaleza, Ceará, Brasil. Rio Grande do Norte, Natal, Brasil.

Oliveira, D. C., et al. (2013). Impacto relatado das alterações bucais na qualidade de vida de adolescentes: Revisão sistemática. Pesquisa brasileira em odontopediatria e clínica integrada. Parana.

Ortiz, F. R., et al. (2020). Gengivitis influences oral health-related quality of life in adolescents: findings from a cohort study. Revista Brasileira de Epidemiologia. Santa Maria - RS, 23.

Perovano, D. G. (2014). Manual de Metodologia Científica. Paraná: Editora Juruá.

Rebouças, A. G., et al. (2017). Fatores individuais associados à má-oclusão em adolescentes. Rev. Ciência \& Saúde Coletiva. Campinas-SP, 22(11).

Sarmento, M. G. S., Santos O. A; \& Lima M. M. (2020). Desafios da educação em saúde bucal na adolescência. REAOdonto 2, ISSN $2674-720$.

Silva, E. K. P., et al. (2018). Saúde bucal de adolescentes rurais quilombolas e não quilombolas: um estudo dos hábitos de higiene e fatores associados. Vitória da Conquista BA Brasil. 
Research, Society and Development, v. 10, n. 15, e290101523217, 2021

(CC BY 4.0) | ISSN 2525-3409 | DOI: http://dx.doi.org/10.33448/rsd-v10i15.23217

Sousa, F. S., et.al. (2020). Persistem iniquidades sociais na distribuição da cárie dentária em adolescentes maranhenses? Contribuições de um estudo de base populacional. São Luís MA Brasil, 2020.

Tomita, N. E., Pernambuco, R.A., Lauris, J.R.P., \& Lopes, E. S. (2001). Educação em Saúde Bucal para Adolescentes: Uso de métodos participativos. Rev. FOB. 9(1/2), 63-69.

Torres-Neto, J. R., et al. (2018). Prevalência e características da doença periodontal em adolescentes: revisão de literatura. Caruaru-PE.

Vilar, M. O. (2020). Prevalência de cárie dentária em crianças em condição de vulnerabilidade social. Revista Multidisciplinar e da Psicologia. Juazeiro do Norte-CE, 14(49), 457. 